\title{
Dynamic analysis of the extended space charge layer using chronopotentiometric measurements
}

\author{
Inhee Cho ${ }^{1,2}$, Hyomin Lee ${ }^{3}$ and Sung Jae Kim ${ }^{2,4,5^{*}}$ (D)
}

\begin{abstract}
In this paper, we experimentally verified the length $\left(L_{E S C}\right)$ and the concentration $\left(C_{E S C}\right)$ of the extended space charge (ESC) layer in front of the electrical double layer (EDL) using the chronopotentiometric measurement and the equivalent circuit model analysis. From the experimentation, the coupled-response of the EDL and the ESC layer was discriminated from the contribution of electro-osmotic flow (EOF). In addition, we derived the potential differences across the ESC $\left(V_{E S C}\right)$ layer using the circuit model of the ICP layer under rigorous consideration of ESC and EDL. As a result, we obtained that $V_{E S C}$ was linearly proportional to the square of the applied current $\left(i_{\text {applied }}\right)$. Hence, $L_{E S C}$ and $C_{E S C}$ were quantitatively provided, where $L_{E S C}$ is linear to the $i_{\text {applied }}$ and $C_{E S C}$ is constant regardless of $i_{\text {applied }}$. Thus, this experimentation could not only clarify an essential ICP theory but also guide in ESC-based applications.
\end{abstract}

Keywords: Ion concentration polarization, Chronopotentiometric measurement, Extended space charge layer

\section{Introduction}

Perm-selective ion selective transportation through an electrochemical nanoporous membrane has been widely utilized for numerous engineering applications such as desalination [1-7], preconcentration [8-17] and energy harvesting [2, 18-22]. In such systems, ion concentration gradients are formed on both sides of the nanoporous membrane, which is called an ion concentration polarization (ICP) phenomenon [23, 24]. Typically, a zone where electrolyte concentration was extremely low was formed at the anodic side of membrane, while the electrolyte concentration significantly increased at the cathodic side of membrane in the case of cation-selective membrane [25]. These zones were called the ion depletion zone and the ion enrichment zone, respectively. In order to characterize these zone, numerous theories and experimentations had been conducted such as the possible

\footnotetext{
*Correspondence: gates@snu.ac.kr

${ }^{2}$ Department of Electrical and Computer Engineering, Seoul National University, Seoul 08826, Republic of Korea

Full list of author information is available at the end of the article
}

overlimiting conductance (OLC) mechanisms by instabilities [26-32], diffusioosmosis [33], electro-osmotic flow (EOF) and surface conduction (SC) [34-38], etc. Most of these studies pointed that all these nonlinear electrokinetic phenomena were stemming from the development of extended space charge (ESC) layer in front of the electrical double layer (EDL) at an overlimiting current regime, predicted by Rubinstein and Zaltzman [39, 40]. Recently, the electrical impedance spectroscopy (EIS) have been proposed to probe the existence of the ESC layer [41-43]. However, the direct confirmation based on AC electric field analysis was lacking, because the electrical response was tightly involved with the couplings of EDL, ESC layer and EOF.

Therefore, in this study, we suggested an experiment and a circuit analysis for obtaining the potential across the ESC layer $\left(V_{E S C}\right)$. First of all, chronopotentiometric measurement (dc bias with constant current) was used to discriminate the electrical response of both the EDL and the ESC layer out of EOF. Furthermore, we proposed the equivalent circuit model of an ion depletion zone reflecting EDL and ESC layer, where each resistor and each 
capacitor are serially connected. From those analyses, we finally obtained the relationship between $V_{E S C}$ and the applied current, which has never been proposed before. Finally, we quantitatively derived the ESC layer information such as the length, the total charge and the concentration. Therefore, this study would be one of essential basis for ICP research not only in fundamental aspect but also various applications based on ICP.

\section{Materials and methods}

\section{Device fabrication}

As shown in Fig. 1a, we fabricated a micro/nanofluidic device consisting of the main microchannel (1 cm length, $100 \mu \mathrm{m}$ height and $15 \mu \mathrm{m}$ depth), the buffer microchannel $(1 \mathrm{~cm}$ length, $100 \mu \mathrm{m}$ height, and $15 \mu \mathrm{m})$ and two side microchannels $(40 \mathrm{~mm}$ length, $15 \mu \mathrm{m}$ height and $15 \mu \mathrm{m}$ depth). For the external hydrodynamic injection, the two side microchannels were tangentially connected to the main microchannel, which is $50 \mu \mathrm{m}$ apart from the end of the main microchannel. The side microchannels on both sides of the main microchannel was installed for easiness of the experiment [44] and preventing ever-increasing ICP layer [45]. By injecting fresh electrolyte solution through the side microchannels, the diffusion length was reduced as an order of a hundred micron, confining the ICP layer as the triangular shape as shown in Fig. 1b, c. The main building block of device were made of a polydimethyl siloxane (PDMS, Sylgard 184 silicone elastomer kit, Dow corning). We followed the general soft-lithographical fabrication method for PDMS [46]. The Nafion nanoporous membrane was patterned on the glass substrate based on the surface patterning method [45, 47]. Simply, Nafion was patterned using a straight microchannel $(200 \mu \mathrm{m}$ width $\times 50 \mu \mathrm{m}$ depth) on a glass side, and the PDMS piece of the main
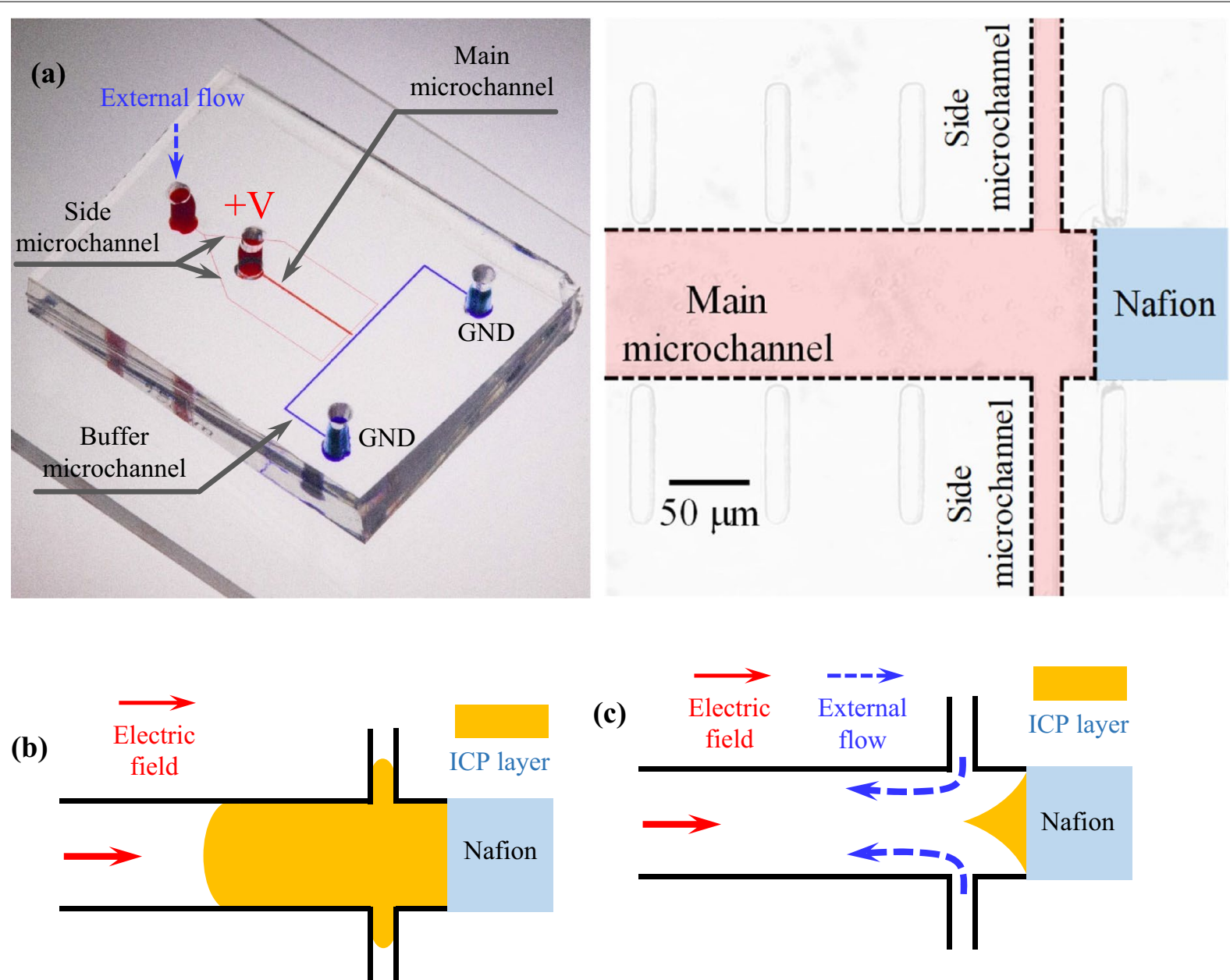

(c)

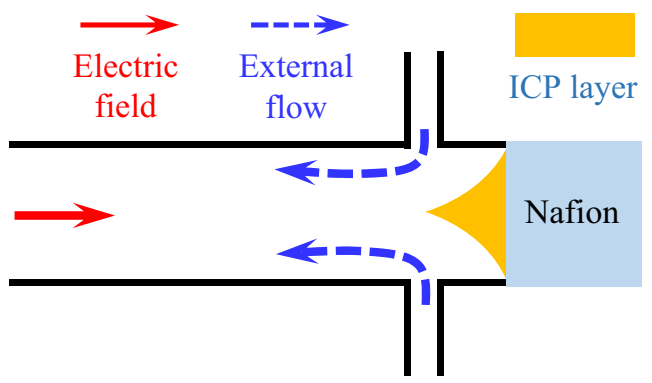

Fig. 1 a Image of micro/nanofluidic device used in this work and the magnified view of the device. Main and buffer microchannel had the dimension of $15 \mu \mathrm{m}$ depth $\times 100 \mu \mathrm{m}$ width and the side microchannel had one of $15 \mu \mathrm{m}$ depth $\times 150 \mu \mathrm{m}$. The ICP layer $\mathbf{b}$ without or $\mathbf{c}$ with external flow from two side microchannels 
microchannel was irreversibly bonded in the middle using a plasma bonder (CuteMP, Femto Science, Korea) to a designated position on top of the Nafion-patterned glass.

\section{Chemical preparation}

Potassium chloride $1 \mathrm{mM}$ solution were used for the experimentation. For tracking the electrokinetic flows and visualizing the ion concentration profile around the ion concentration polarization (ICP) layer, the negatively charged particle $(d=0.2 \mu \mathrm{m}$, Invitrogen) and the fluorescent dye (Alexa488, Sigma Aldrich) were mixed in the prepared solution $[33,37,48]$.

\section{Experimental setup}

From the two side microchannels, we pumped the prepared solution with the volume rate, $20 \mathrm{~nL} / \mathrm{min}$ using a syringe pump (PHD2000, Harvard apparatus) for $30 \mathrm{~min}$ until the injected flows were stabilized at the main microchannel. Then, we applied the external current source through the reservoir of the main microchannel utilizing the source measure unit (SMU 236, Keithley) while the two reservoirs of the buffer microchannel were grounded. Note that the reservoir of the two side microchannels were electrically floated during ICP. With a customized LabView program, we performed four experimentations as followed: (1) the chronopotentiometric measurement $(V-t)$ from 1 to $30 \mathrm{nA}$ with an $1 \mathrm{nA}$ interval for each $3 \mathrm{~min}$, (2) the chronoamperometric measurement $(I-t)$ from 0.3 to $9.9 \mathrm{~V}$ with an $0.3 \mathrm{~V}$ interval for each $3 \mathrm{~min}$, (3) the voltage-current $(V-I)$ responses from 0 to $30 \mathrm{nA}$ with a step current $1 \mathrm{nA}$ for every $60 \mathrm{~s}$ per step and (4) the current-voltage $(I-V)$ responses from 0 to $9.9 \mathrm{~V}$ with a step voltage $0.3 \mathrm{~V}$ for every $60 \mathrm{~s}$ per step. In order to capture the optical image of an ICP layer, we used a CCD camera (DP73, Olympus) and the image was obtained through the commercial software program (CellSens, Olympus).

\section{Results and discussions}

\section{Chronopotentiometric measurement}

Figure 2 showed the representing chronopotentiometric measurement of the ICP system, where the red line and the blue line indicated the electrical response at both the overlimiting current regime and the ohmic current regime, respectively. Previous studies neglected the voltage behavior at the ohmic current regime, while they described the voltage behavior at the overlimiting current regime as: (1) The initial voltage value was ohmic voltage which was subject to the electrodialysis system. (2) The sharp voltage hop (1st hop) appeared and the voltage value depended on the type of membrane. (3) A linear voltage growth (2nd hop) regime was followed, where

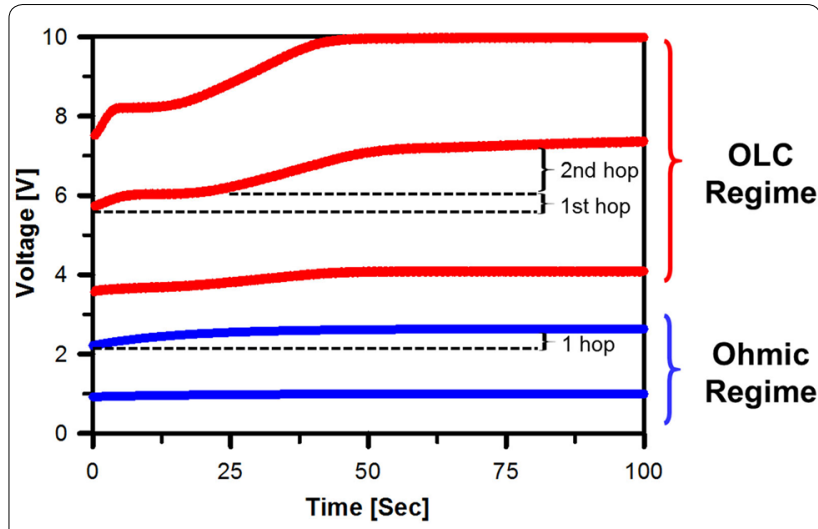

Fig. 2 Chronopotentiometric measurements for all current values through ICP layer

the electroconvection initiated at this time, and then (4) the voltage value was saturated as the microvortices saturated both the size and speed $[49,50]$ However, the aforementioned steps were insufficient to explain the voltage behavior in chronopotentiometry since the ICP layer model was missing. Furthermore, the internal structures inside ion depletion zone has never been suggested as an electrokinetic circuit model. Thus, we would introduce a unified equivalent circuit model including EDL and ESC as well as 2nd EOF in the following section.

Especially at the OLC regime, the voltage responses during the chronopotentiometric measurement showed the two voltage hops $\left(V_{1 s t}\right.$ and $\left.V_{2 n d}\right)$ as shown in Fig. 3a. When the current was applied at $t=0$ (sec) from the main microchannel, the $V_{1 s t}$ was followed due to the capacitance of both the ESC and the EDL, which the corresponding image and the circuit was shown in image i) in Fig. 3a and inset of in Fig. 3b, respectively. When the EOF was generated at $t=15 \mathrm{~s}$, the voltage was increasing until the EOF size saturated at $t>50 \mathrm{~s}$ with the value $V_{2 n d} \sim i_{\text {applied }}$ as shown in image ii) in Fig. 3a [50]. In this experimentation, we applied the various current values from $12 \mathrm{nA}$ to $30 \mathrm{nA}$ so that we can obtain the $V_{1 s t}-i_{a p-}$ plied relations as shown in Fig. 3b. Note that the $V_{1 s t}$ is not linear to the $i_{\text {applied }}$ indicating that the ohm's law is not valid due to the appearance of the ESC layer as expected by Rubinstein and Zaltzman [40].

\section{Equivalent electrokinetic circuit model of the ICP layer} At the charged membrane surface, the EDL was composed of both resistor $\left(R_{E D L}\right)$ and capacitor $\left(C_{E D L}\right)$ in parallel and they were connected in series to the diffuse layer resistor $\left(R_{\text {bulk }}\right)$ as in Fig. 4a. This simple circuit coincided with the voltage-time behavior in the ohmic regime, which showed the gentle slope and the slight voltage hop as in Fig. 2. Once the current was applied exceeding 

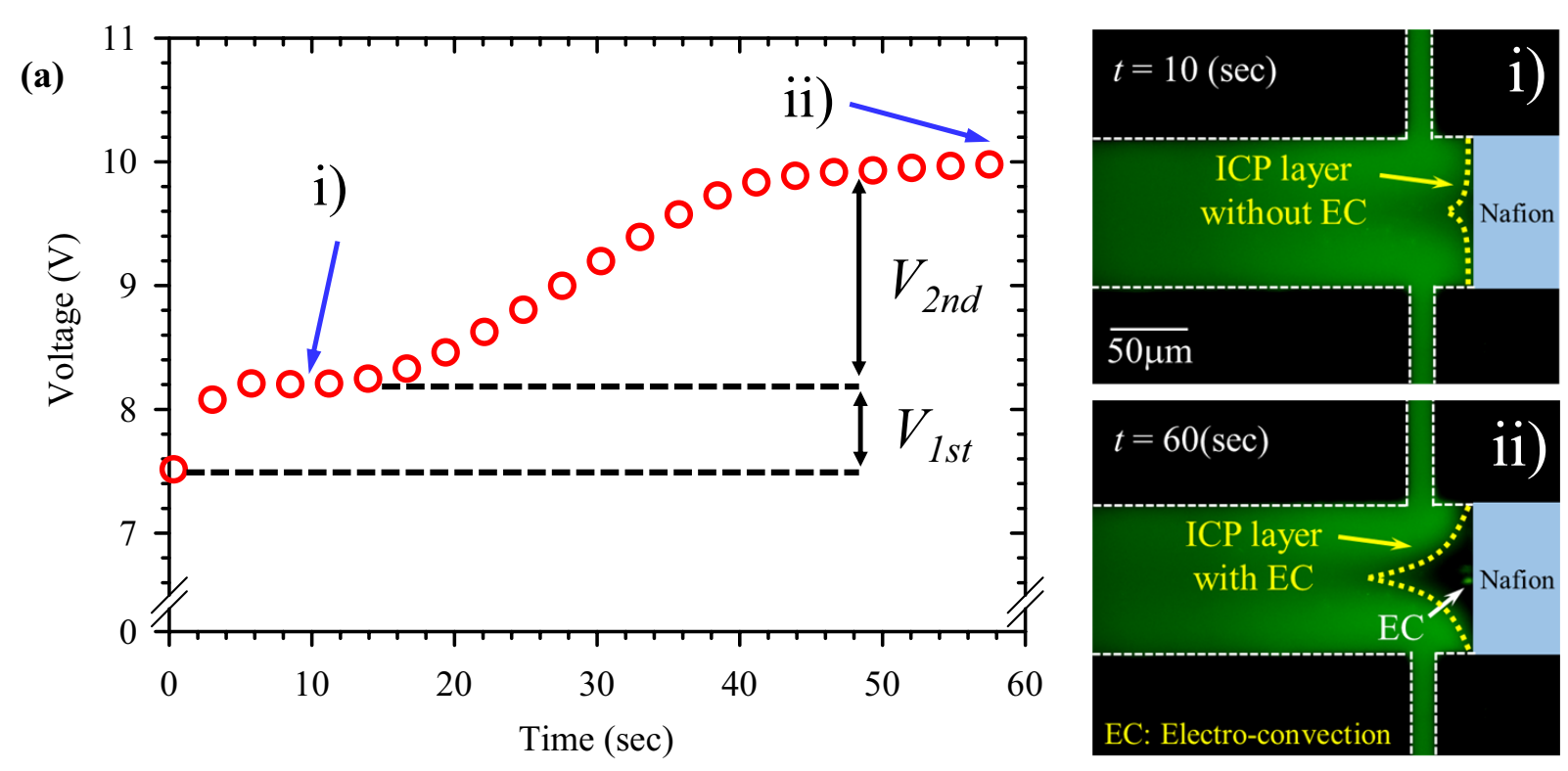

(b)

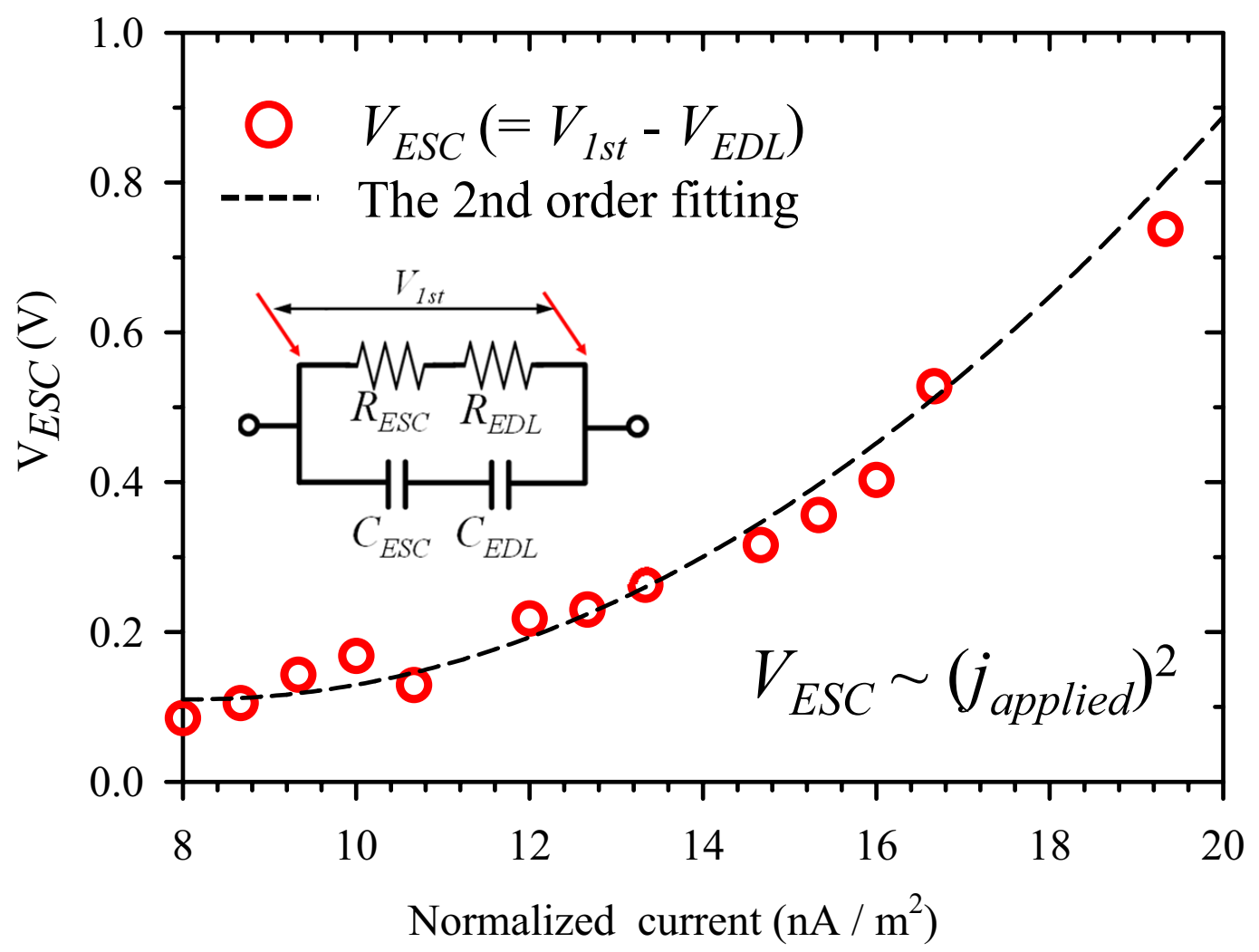

Fig. 3 a The result of chronopotentiometric measurement at $29 \mathrm{nA}$ (in the regime of OLC regime) and the corresponding images at i) $t=10 \mathrm{~s}$ and ii) $t=60 \mathrm{~s}$, respectively. $\mathbf{b}$ The $V_{1 s t} t_{\text {applied }}$ relations and the equivalent circuit model of the ICP layer at inset of the graph 
limiting current, the ESC layer grew between the EDL and the diffuse layer, where both resistor $\left(R_{E S C}\right)$ and capacitor $\left(C_{E S C}\right)$ should be additionally employed as in Fig. 4b. This electrical circuit model affected the total RC delay time, converting the gentle slope at ohmic current regime into the sharp one at overlimiting current regime.

Normally, time-varying voltage responses existed where the resistance and the capacitance are parallel in the circuit model. Considering that bulk solution was regarded to the quasi-neutral regions, one can ignore the capacitance. In the meantime, the sufficient charge carriers existed inside the electrical double layer (EDL) for compensating the charged surface (e.g. Nafion), thus one should consider the capacitance of the EDL as well as the resistance of one. This means that the voltage responses should be divided into the constant term (for diffusion layer) and the time-varying one (for EDL) as follows:

$$
V(t)=V_{0}+V_{E D L} \exp \left(-\frac{t}{\tau_{E D L}}\right)
$$

where $V_{0}$ is the potential of diffuse layer, $V_{E D L}$ the potential of EDL and the $\tau_{E D L}$ is the RC delay time $\left(\tau_{E D L}=R_{E D L}\right.$ $C_{E D L}$ in the circuit model). As shown in Additional file 1: Figure S4, the collapsed data of the $V_{E D L}$ has the linear relations to the applied current density, which lead to the constant resistance values $\left(R_{E D L}-V_{E D L} / I\right)$ as $3 \mathrm{M} \Omega$. Each component has the value $240 \pm 42 \mathrm{M} \Omega$ (for $R_{b u l k}$ ), $3 \pm 0.7 \mathrm{M} \Omega$ (for $\left.R_{E D L}\right), 6 \pm 1.2 \mu \mathrm{F}$ (for $\left.C_{E D L}\right), 1.09\left(I-I_{\text {lim }}\right.$ ) $\mathrm{M} \Omega$ (for $R_{E S C}$ ) and $2.23\left(I-I_{\text {lim }}\right)^{-1} \mu \mathrm{F}$ (for $C_{E S C}$ ), respectively. The simple calculation result and the derivations was introduced in supporting materials (Additional file 1: Table S1, Figure S4).

Valenca and co-workers reported that the microvortices by ICP induced the potential difference at $V_{2 n d}$ in the EC dominant regime [50]. This indicated that, in a certain overlimiting current value $I_{O L C}>I_{\text {lim }}$, one can estimate the point conductance at $I_{O L C}$ with a simple calculation as $\sigma_{O L C}=I / V_{2 n d}$. We also confirmed the conductivities in EOF regime, where the applied current is ranging from $12 \mathrm{nA}$ to $29 \mathrm{nA}$, leading to OLC by EOF as the constant value of $0.21 \mathrm{nS}$ in our system. Note that the experimental results and the set of data were provided in supporting materials (Additional file 1: Figure S2). In addition, critical time $\left(T_{c}\right)$ that initiate the EOF has the relation of the OLC conductance $\left(\sigma_{O L C}\right)$ and its time-derivative one $\left(\partial \sigma_{O L C} / \partial t\right)$. This means that $T_{c}$ is also subject to the $V_{2 n d}$ and its time-derivative one $\left(\partial V_{2 n d} / \partial t\right)$. The scaling was developed and quantified in supporting materials (Additional file 1: Figure S3).

\section{The length $\left(L_{E S C}\right)$ and the concentration ( $\left.c_{E S C}\right)$ of the ESC layer}

At the ESC layer, the dimensionless length $\left(\tilde{L}_{E S C}\right.$, normalized by diffusion length) should be $0.5\left(3 \varepsilon V_{E S C}\right)\left({ }^{2 / 3}\right)$ $(j)(-1 / 3)$, the total space charge density $\left(\Sigma_{E S C}\right)$ should be $\left.\varepsilon^{4 / 3}\right)\left(2 j V_{E S C}\right)\left({ }^{1 / 3}\right)$ and the concentration $\left(c_{E S C}\right)$ should be $0.69\left(\varepsilon^{2} \psi_{E S C}\left({ }^{-1}\right) j^{2}\right)\left({ }^{1 / 3}\right)[51,52]$. Here $\varepsilon$ is the dimensionless Debye length; $\psi_{E S C}$ is the dimensionless electric potential (normalized by the thermal potential $R T / F$ ) and the $j$ is the dimensionless applied current density (normalized by the cross-sectional are of microchannel). In our system, the diffusion length was $100 \mu \mathrm{m}, \varepsilon$ was $4.26 \times 10^{-4}$ and $\psi_{E S C} \approx\left(7.89 \times 10^{-2}\right) \times j^{2}$, leading to $L_{E S C} \approx 132 \times j(\mathrm{~nm})$, $\Sigma_{E S C} \approx 0.225 \times j\left(\mu \mathrm{q} / \mathrm{m}^{3}\right)$ and $c_{E S C} \approx 2.30(\mu \mathrm{M})$, respectively, leading to the conclusion of $L_{E S C} \sim j_{\text {applied }}, \Sigma_{E S C} \sim j_{a p-}$ plied and $c_{E S C} \sim$ constant inside the ESC layer as shown in Fig. 5.

\section{Conclusions}

Recent experiments have been conducted for probing the space charge at the micro- and nano-channel interface device using electrical impedance spectroscopy (EIS),

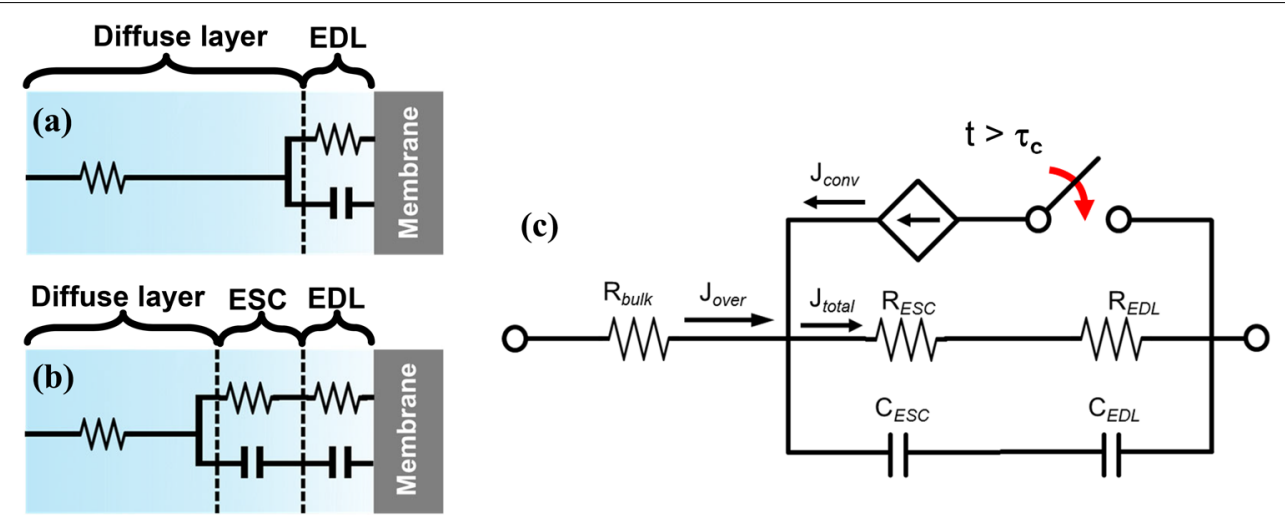

Fig. 4 Schematics of equivalent circuit of ICP layer at $\mathbf{a}$ ohmic current regime and $\mathbf{b}$ overlimiting current regime. $\mathbf{c}$ A unified equivalent electrokinetic circuit model of ICP layer considering EDL, ESC and 2nd EOF 


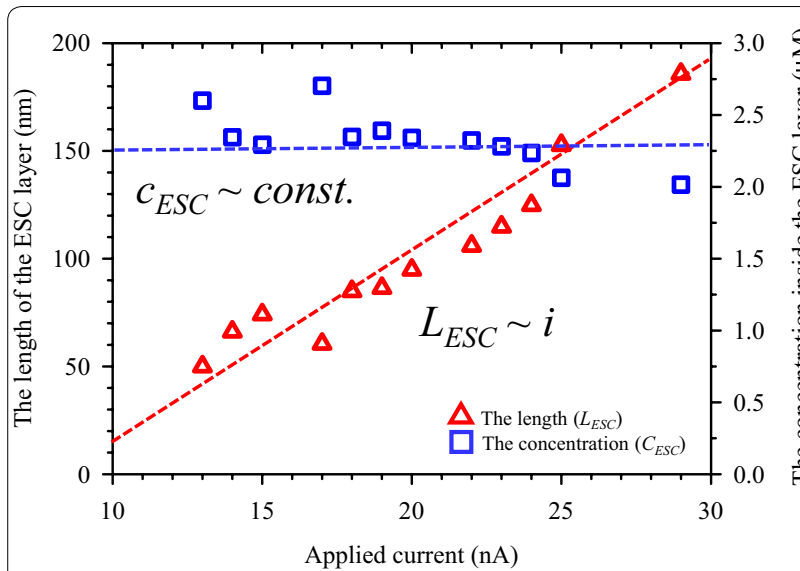

Fig. 5 The length $\left(L_{E S C}\right)$ and the concentration $\left(C_{E S C}\right)$ of the ESC layer, respectively

employing a conventional equivalent circuit model. However, those literatures revealed out that the EIS method hardly determined the ESC layer response since the multiple electrokinetic responses were tightly coupled during ICP. For example, Yossifon and co-workers probed the diffusion layer(DL) and the electrical double layer(EDL) using EIS at the micro- and nano-channel systems [43]. They found out the detailed components of the EDL by separating the electrode-fluidic interface and microchannel-nanochannel interface. From this experiment, they clearly captured the resistances and the capacitances at both EDL for satisfying the theoretical calculations. However, this demonstration fails to present ESC layer responses at the higher voltage because of the coupling effect where electroconvective flows were involved, thereby arousing another issue for differentiating them, individually. Thus, we emphasized that this equivalent circuit model, for the first time, reflected EDL and ESC layer as well as the convective flows using the micro-/ nano-fluidic systems.

In this paper, we experimentally investigated the ESC layer using chronopotentiometric measurement and the unified equivalent electrokinetic circuit model of internal ICP structure with the consideration of EDL, ESC and 2nd EOF. Each electrical component such as two resistors, two capacitors and dependent current source were included in the new model, confirming the voltage responses in chronopotentiometric measurement. From our rigorous experimentation, we obtained the relationship between the potential across the ESC layer and the applied current, $V_{E S C}-i_{\text {apptied }}^{2}$ Furmore, we quantitatively provided the $L_{E S C}-j_{\text {applied }}$ and the $c_{E S C} \sim$ constant. Therefore, all this experimental verification of the ESC layer could lead to the further development of ICP theory as well as the ESC/ICP layer related applications.

\section{Supplementary information}

Supplementary information accompanies this paper at https://doi. org/10.1186/s40486-020-00112-1.

\begin{abstract}
Additional file 1: Figure S1. In order to obtain the limiting current values, we conducted the voltage-sweeping method in our systems. Under the 20nL/min flows was applied near the Nafion membrane, the limiting current value reaches $12(\mathrm{nA})$. Figure $\mathbf{S 2}$. The $V_{2 n d}$ from the measurement has been obtained with the applied current, I. This result showed that the slope of $V_{2 n d}-1$, which is the overlimiting conductance (OLC) by electroosmotic flows (EOF) have the constant values as $0.21 \mathrm{nS}$. Figure S3.

The onset time $\left(\tau_{C}\right)$ of electro-convective flows was obtained from the chronopotentiometric measurement. The $\tau_{C}$ values are between 10 and 30 , which result is coincided our scaling theory, $\tau_{c}-\mathrm{O}\left(10^{1}\right)$. Figure $\mathbf{S} 4$. The $V_{15 t}$ which was time-varying potential reflected by the electrical double layer, was obtained from the chronopotentiometric measurement. From this result, the resistance can be calculated by Ohm's law $\left(R_{E D L}=V_{E D L} / l\right)$. (b) The RC delay time caused by the electrical double layer was collected in ohmic current regime. The RC delay times in our experiments were almost constant as the value of $18 \mathrm{~s}$ regardless of the applied current. From this result, the capacitance can be calculated by $\left(C_{E D L}=t_{E D L} / R_{E D L}\right)$. Table $\mathbf{S 1}$. The electrical components of the equivalent circuit model were calculated by simple calculation. Note that $R_{E D L}$ and $C_{E D L}$ remains same regardless of the applied current (I), while $R_{E S C}$ and $C_{E S C}$ are linearly proportional to the current values $\left(I-I_{\text {lim }}\right)$, where $I_{\text {lim }}$ is the limiting current values.
\end{abstract}

\section{Abbreviations}

ICP: Ion concentration polarization; EDL: Electrical double layer; ESC: Extended space charge; EOF: Electroosmotic flow; SC: Surface conduction; OLC: Overlimiting conductance; PDMS: Poly-dimethyl siloxane; EIS: Electrical impedance spectroscopy.

\section{Acknowledgements}

All authors acknowledged the supports from BK21 Plus program of the Creative Research Engineer Development IT, Seoul National University.

\section{Authors' contributions}

IC conducted the main experiment. HL advised circuit modeling. SJK supervised the project. All authors read and approved the final manuscript.

\section{Funding}

This work is supported by the Basic Research Laboratory Project (NRF2018R1A4A1022513) and Mid-Career Project (NRF-2020R1A2C3006162) by the Ministry of Science and ICT. I. Cho was supported by the National Research Foundation of Korea (NRF) grant funded by the Ministry of Science and ICT (NRF-2020R1F1A1072960).

\section{Availability of data and materials}

All data generated or analyzed during this study are included in this published article.

\section{Competing interests}

The authors declare no competing interests (both financial and non-financial).

\section{Author details}

${ }^{1}$ Korea-Russia Innovation Center, Korea Institute of Industrial Technology, Incheon 22004, Republic of Korea. ${ }^{2}$ Department of Electrical and Computer Engineering, Seoul National University, Seoul 08826, Republic of Korea. ${ }^{3}$ Department of Chemical and Biological Engineering, Jeju National University, Jeju 63243, Republic of Korea. ${ }^{4}$ Inter-university Semiconductor Research Center, Seoul National University, Seoul 08826, South Korea. ${ }^{5}$ Nano Systems Institute, Seoul National University, Seoul 08826, South Korea.

Received: 2 March 2020 Accepted: 28 May 2020

Published online: 05 June 2020 


\section{References}

1. Kim SJ, Ko SH, Kang KH, Han J (2010) Direct seawater desalination by ion concentration polarization. Nat Nanotech 5:297-301

2. Kim B, Choi S, Pham VS, Kwak R, Han J (2017) Energy efficiency enhancement of electromembrane desalination systems by local flow redistribution optimized for the asymmetry of cation/anion diffusivity. J Membr Sci 524:280-287

3. Kim B, Kwak R, Kwon HJ, Pham VS, Kim M, Al-Anzi B, Lim G, Han J (2016) Purification of high salinity brine by multi-stage ion concentration polarization desalination. Sci Rep 6:31850

4. Kwak R, Kim SJ, Han J (2011) Continuous-flow biomolecule and cell concentrator by ion concentration polarization. Anal Chem 83:7348-7355

5. Knust KN, Hlushkou D, Anand RK, Tallarek U, Crooks RM (2013) Electrochemically mediated seawater desalination. Angewandte chemie Int Edition 52:8107-8110

6. Deng D, Aouad W, Braff WA, Schlumpberger S, Suss ME, Bazant MZ (2015) Water purification by shock electrodialysis: deionization, filtration, separation, and disinfection. Desalination 357:77-83

7. Park S, Jung Y, Son SY, Cho I, Cho Y, Lee H, Kim H-Y, Kim SJ (2016) Capillarity ion concentration polarization as spontaneous desalting mechanism. Nat Commun 7:11223

8. Fu LM, Hou HH, Chiu PH, Yang RJ (2018) Sample preconcentration from dilute solutions on micro/nanofluidic platforms: a review. Electrophoresis 39:289-310

9. Kim SJ, Song Y-A, Han J (2010) Nanofluidic concentration devices for biomolecules utilizing ion concentration polarization: theory, fabrication, and application. Chem Soc Rev 39:912-922

10. Son SY, Lee S, Lee H, Kim SJ (2016) Engineered nanofluidic preconcentration devices by ion concentration polarization. BioChip J 10:251-261

11. Choi J et al (2015) Selective preconcentration and online collection of charged molecules using ion concentration polarization. RSC Adv 5:66178-66184

12. Lee $\mathrm{H}$ et al (2018) dCas9-mediated nanoelectrokinetic direct detection of target gene for liquid biopsy. Nano Lett 18:7642-7650

13. Baek S, Choi J, Son SY, Kim J, Hong S, Kim HC, Chae J-H, Lee H, Kim SJ (2019) Dynamics of driftless preconcentration using ion concentration polarization leveraged by convection and diffusion. Lab Chip 19:3190-3199

14. Lee S, Park S, Kim W, Moon S, Kim H-Y, Lee H, Kim SJ (2019) Nanoelectrokinetic bufferchannel-less radial preconcentrator and online extractor by tunable ion depletion layer. Biomicrofluidics 13:034113

15. Ko SH, Song YA, Kim SJ, Kim M, Han J, Kang KH (2012) Nanofluidic preconcentration device in a straight microchannel using ion concentration polarization. Lab Chip 12:4472-4482

16. Cheow LF, Sarkar A, Kolitz S, Lauffenburger D, Han J (2014) Detecting kinase activities from single cell lysate using concentration-enhanced mobility shift assay. Anal Chem 86:7455-7462

17. Chen C-H, Sakar A, Song Y-A, Miller MA, Kim SJ, Griffith LG, Lauffenburger DA, Han J (2011) Enhancing protease activity assay in droplet-based microfluidics using a biomolecule concentrator. J Am Chem Soc 133:10368-10371

18. Subramani A, Badruzzaman M, Oppenheimer J, Jacangelo JG (2011) Energy minimization strategies and renewable energy utilization for desalination: a review. Water Res 45:1907-1920

19. Kjeang E, Djilali N, Sinton D (2009) Microfluidic fuel cells: a review. J Power Sources 186:353

20. Song Y-A, Batista C, Sarpeshkar R, Han J (2008) In-plane integration of ionselective membrane in microfluidic PEM fuel cell by micro flow surface patterning. J Power Sources 183:674-677

21. Costa P, Bosio B (2008) Identification problems and analysis of the limit current in fuel cells. J Power Sources 185:1141-1146

22. St-Pierre J, Wetton B, Kim G-S, Promislow K (2007) Limiting current operation of proton exchange membrane fuel cells. J Electrochem Soc 154:B186-B193

23. Kim SJ, Wang Y-C, Lee JH, Jang H, Han J (2007) Concentration polarization and nonlinear electrokinetic flow near nanofluidic channel. Phys Rev Lett 99:044501

24. Zangle TA, Mani A, Santiago JG (2010) Theory and experiments of concentration polarization and ion focusing at microchannel and nanochannel interfaces. Chem Soc Rev 39:1014-1035
25. Pu Q Yun J, Temkin H, Liu S (2004) Ion-enrichment and ion-depletion effect of nanochannel structures. Nano Lett 4:1099-1103

26. Magnico $P(2018)$ Spatial distribution of mechanical forces and ionic flux in electro-kinetic instability near a permselective membrane. Phys Fluids 30:014101

27. Andersen MB, Wang KM, Schiffbauer J, Mani A (2017) Confinement effects on electroconvective instability. Electrophoresis 38:702-711

28. Rubinstein I, Zaltzman B (2015) Equilibrium electroconvective instability. Phys Rev Lett 114:114502

29. Druzgalski C, Andersen MB, Mani A (2013) Direct numerical simulation of electroconvective instability and hydrodynamic chaos near an ionselective surface. Phys Fluids 25:110804

30. Kim SJ, Ko SH, Kwak R, Posner JD, Kang KH, Han J (2012) Multi-vortical flow inducing electrokinetic instability in ion concentration polarization layer. Nanoscale 4:7406-7410

31. Andersen MB, van Soestbergen M, Mani A, Bruus $H$, Biesheuvel PM Bazant MZ (2012) Current-induced membrane discharge. Phy Rev Lett 109:108301

32. Demekhin E, Shelistov V, Polyanskikh S (2011) Linear and nonlinear evolution and diffusion layer selection in electrokinetic instability. Phys Rev E 84:036318

33. Cho I, Kim W, Kim J, Kim H-Y, Lee H, Kim SJ (2016) Non-negligible diffusio-osmosis inside an ion concentration polarization layer. Phys Rev Lett 116:254501

34. Dydek EV, Zaltzman B, Rubinstein I, Deng DS, Mani A, Bazant MZ (2011) Overlimiting current in a microchannel. Phys Rev Lett 107:118301

35. Nam S, Cho I, Heo J, Lim G, Bazant MZ, Moon DJ, Sung GY, Kim SJ (2015) Experimental verification of overlimiting current by surface conduction and electro-osmotic flow in microchannels. Phys Rev Lett 114:114501

36. Sohn S, Cho I, Kwon S, Lee H, Kim SJ (2018) Surface conduction in a microchannel. Langmuir 34:7916-7921

37. Huh K, Yang S-Y, Park JS, Lee JA, Lee H, Kim SJ (2020) Surface conduction and electroosmotic flow around charged dielectric pillar arrays in microchannels. Lab Chip 20:675-686

38. Kim K, Kim W, Lee H, Kim SJ (2017) Stabilization of ion concentration polarization layer using micro fin structure for high-throughput applications. Nanoscale 9:3466-3475

39. Zaltzman B, Rubinstein I (2007) Electro-osmotic slip and electroconvec tive instability. J Fluid Mech 579:173-226

40. Rubinstein I, Zaltzman B (2010) Extended space charge in concentration polarization. Adv Colloid Interface Sci 159:117-129

41. Park S, Yossifon G (2016) Induced-charge electrokinetics, bipolar current, and concentration polarization in a microchannel-Nafionmembrane system. Phys Rev E 93:062614

42. Green Y, Shloush S, Yossifon G (2014) Effect of geometry on concentration polarization in realistic heterogeneous permselective systems. Phys Rev E (Statistical Nonlinear and Soft Matter Physics) 89:043015

43. Schiffbauer J, Park S, Yossifon G (2013) Electrical impedance spectroscopy of microchannel-nanochannel interface devices. Phys Rev Lett 110:204504

44. Kim J, Kim H-Y, Lee H, Kim SJ (2016) Pseudo 1-D micro/nanofluidic device for exact electrokinetic responses. Langmuir 32:6478-6485

45. Cho I, Sung G, Kim SJ (2014) Overlimiting current through ion concentration polarization layer: hydrodynamic convection effects. Nanoscale 6:4620-4626

46. Duffy DC, McDonald JC, Schueller OJA, Whitesides GM (1998) Rapid prototyping of microfluidic systems in poly(dimethylsiloxane). Anal Chem 70:4974-4984

47. Lee JH, Song Y-A, Han J (2008) Multiplexed proteomic sample preconcentration device using surface-patterned ion-selective membrane. Lab Chip 8:596-601

48. Lee H, Alizadeh S, Kim TJ, Park S-M, Soh HT, Mani A, Kim SJ (2019) Overlimiting current in non-uniform arrays of microchannels. arXiv preprint arXiv:1910.09546

49. Krol J, Wessling M, Strathmann H (1999) Concentration polarization with monopolar ion exchange membranes: current-voltage curves and water dissociation. J Membr Sci 162:145-154

50. de Valença JC, Wagterveld RM, Lammertink RG, Tsai PA (2015) Dynamics of microvortices induced by ion concentration polarization. Phys Rev $\mathrm{E}$ 92:031003 
51. Rubinstein I, Zaltzman B (2010) Dynamics of extended space charge in concentration polarization. Phys Rev E 81:061502

52. Khair AS (2011) Concentration polarization and second-kind electrokinetic instability at an ion-selective surface admitting normal flow. Phys Fluids 23:072003

\section{Publisher's Note}

Springer Nature remains neutral with regard to jurisdictional claims in published maps and institutional affiliations.
Submit your manuscript to a SpringerOpen ${ }^{\odot}$ journal and benefit from:

- Convenient online submission

- Rigorous peer review

- Open access: articles freely available online

- High visibility within the field

- Retaining the copyright to your article

Submit your next manuscript at $\boldsymbol{\nabla}$ springeropen.com 